\title{
New travelling wave solutions of the Porous-Fisher model with a moving boundary
}

\author{
Nabil T. Fadai*, Matthew J. Simpson \\ School of Mathematical Sciences, Queensland University of Technology, Brisbane, \\ Queensland 4001, Australia. \\ E-mail: *nabil.fadai@qut.edu.au
}

\begin{abstract}
We examine travelling wave solutions of the Porous-Fisher model, $\partial_{t} u(x, t)=u(x, t)[1-u(x, t)]+\partial_{x}\left[u(x, t) \partial_{x} u(x, t)\right]$, with a Stefan-like condition at the moving front, $x=L(t)$. Travelling wave solutions of this model have several novel characteristics. These travelling wave solutions: (i) move with a speed that is slower than the more standard Porous-Fisher model, $c<1 / \sqrt{2}$; (ii) never lead to population extinction; (iii) have compact support and a well-defined moving front, and (iv) the travelling wave profiles have an infinite slope at the moving front. Using asymptotic analysis in two distinct parameter regimes, $c \rightarrow 0^{+}$and $c \rightarrow 1 / \sqrt{2}-$, we obtain closed-form mathematical expressions for the travelling wave shape and speed. These approximations compare well with numerical solutions of the full problem.
\end{abstract}

Keywords: Fisher's equation, nonlinear degenerate diffusion, Stefan condition, moving boundary problem

Submitted to: J. Phys. A: Math. Theor.

\section{Introduction}

Travelling waves arise in many fields, including ecology [1-4], cell biology [5-11], and industrial applications involving heat and mass transfer [12 15]. Such processes are often modelled using reaction-diffusion equations and, depending on the choice of reaction and diffusion terms, three broad classes of monotone travelling waves are commonly reported (Figure 1). The most commonly-observed travelling wave is a smooth front (Figure 1a), whereby the concentration, $u(x, t)$, is a monotone decreasing function decaying to zero as $x \rightarrow \infty$. For example, travelling wave solutions of the Fisher-KPP model [1, 2, 7],

$$
\partial_{t} u(x, t)=u(x, t)[1-u(x, t)]+\partial_{x x} u(x, t), \quad-\infty<x<\infty,
$$

are smooth. Unfortunately, smooth fronts do not have compact support, which makes defining the "edge" of the moving front ambiguous [8, 9, 16]. This feature of the FisherKPP model means that it can be hard to apply to practical problems, such as cell invasion [7 9], where well-defined fronts are often observed. 

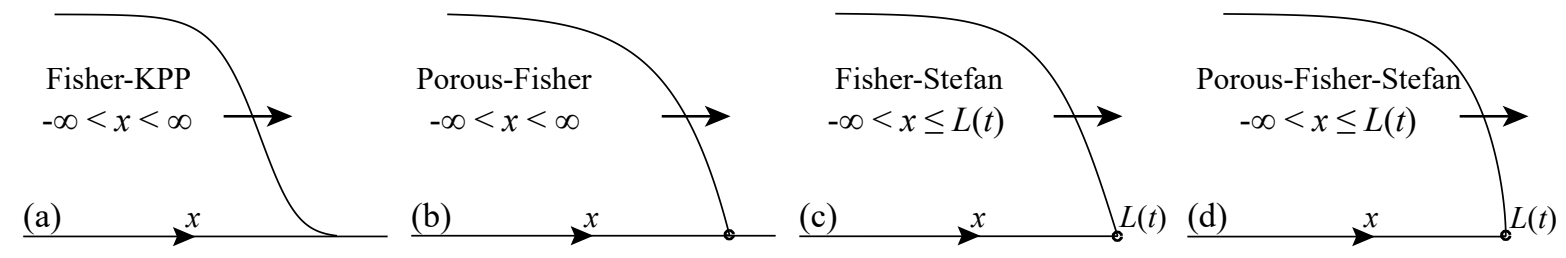

Figure 1. Schematic representation of various monotone travelling waves.

To obtain travelling wave solutiuons with a well-defined front, two main modifications of the Fisher-KPP model have been proposed. The first modification involves incorporating nonlinear degenerate diffusion, whereby the concentration flux is generalized to $-D(u) \partial_{x} u$, with $D(0)=0$. One common choice of degenerate diffusivity is $D(u) \equiv u$, which leads to the Porous-Fisher model [10, 11, 17, 23]:

$$
\partial_{t} u(x, t)=u(x, t)[1-u(x, t)]+\partial_{x}\left[u(x, t) \partial_{x} u(x, t)\right], \quad-\infty<x<\infty .
$$

The Porous-Fisher model supports travelling wave solutions that move with speed $c \geq c_{\min }$, where $c_{\min }=1 / \sqrt{2}[1,19,20,24]$. Phase plane analysis shows that travelling wave solutions of the Porous-Fisher model are sharp-fronted, with compact support, when $c=1 / \sqrt{2}[1,20,24]$. In contrast, travelling wave solutions of the Porous-Fisher model with $c>1 / \sqrt{2}$ are smooth and do not have compact support [1, 24]. Interestingly, travelling wave solutions of the Porous-Fisher model with $c<1 / \sqrt{2}$ have never been reported.

The second modification of the Fisher-KPP model that leads to a well-defined front is to maintain the use of linear diffusion, but to incorporate a moving boundary condition so that we consider the Fisher-KPP model on $-\infty<x<L(t)$ [4, 7, 25]. This second modification of the Fisher-KPP model has been called the Fisher-Stefan model [7, 25], which incorporates a Stefan-like condition at the moving boundary, $L(t)$, to relate the concentration flux, $-\partial_{x} u(x, t)$, with the speed of the moving boundary. One of the limitations of the Fisher-Stefan model is that this model allows populations to become extinct [7, 25], since there is an outward flux of at the leading edge, $x=L(t)$. While the Fisher-Stefan model has the advantage that it can lead to sharp-fronted travelling waves, the physical or biological explanation of the outward flux at $x=L(t)$ is not obvious.

Both the Porous-Fisher and the Fisher-Stefan models have the advantage that they lead to travelling wave solutions with a well-defined front (Figure 1 b,c). While these two modifications of the Fisher-KPP model have been considered previously, the extension of combining nonlinear degenerate diffusion with a moving boundary condition has yet to be considered. We refer to this combination of the Porous-Fisher model with a moving boundary condition as the Porous-Fisher-Stefan model, which we define as the Porous-Fisher equation (2) on $x \in(-\infty, L(t)$ ] with a Stefan-like condition stating that the speed of the moving front, $\mathrm{d} L(t) / \mathrm{d} t$, is proportional to the nonlinear concentration flux, $-u(x, t) \partial_{x} u(x, t)$, with constant of proportionality $\kappa>0$. Unlike the Fisher-Stefan model [7, 25], the incorporation of nonlinear degenerate diffusion in the Porous-Fisher- 
Stefan model prevents $u(x, t)$ from being driven to extinction. Interestingly, preliminary numerical solutions of the Porous-Fisher-Stefan model suggest that travelling wave solutions exist with $0 \leq c<1 / \sqrt{2}$ and that these sharp-fronted travelling waves have infinite slope at $x=L(t)$ (Figure $1 \mathrm{~d}$ ). Neither of these properties have been reported or analyzed previously.

In this work, we examine sharp-fronted travelling waves that arise in the PorousFisher-Stefan model. By transforming this model into travelling wave coordinates, we examine the phase plane for various regimes of the wave speed $c$. Using asymptotic analysis, we obtain approximations of the resulting phase plane trajectories when $c \ll 1$ and when $c$ is close to the critical wave speed $1 / \sqrt{2}$. Additionally, we determine the relationship between the travelling wave speed $c$ and the Stefan parameter $\kappa$. In doing so, we determine an approximate form of the travelling wave front that matches numericallycomputed travelling wave solutions with high accuracy.

\section{Travelling waves in the Porous-Fisher-Stefan model}

We consider the non-dimensional Porous-Fisher model, describing the concentration $u(x, t) \in[0,1]$ with a Stefan-like condition at the moving boundary $x=L(t)$ :

$$
\begin{gathered}
\partial_{t} u(x, t)=u(x, t)[1-u(x, t)]+\partial_{x}\left[u(x, t) \partial_{x} u(x, t)\right], \quad-\infty<x<L(t), \\
\lim _{x \rightarrow-\infty} u(x, t)=1, \quad u(L(t), t)=0, \\
\frac{\mathrm{d} L(t)}{\mathrm{d} t}=-\left.\kappa u(x, t) \partial_{x} u(x, t)\right|_{x \rightarrow L(t)^{-}}, \quad L(0)=L_{0} .
\end{gathered}
$$

The Stefan-like condition relates the speed of the moving front, $\mathrm{d} L(t) / \mathrm{d} t$, to the concentration flux, $-u(x, t) \partial_{x} u(x, t)$, via the constant $\kappa>0$. Preliminary numerical solutions of (3) - (5) suggest that travelling waves exist and that these waves move with speed $0 \leq c<1 / \sqrt{2}$ with infinite gradient at $x=L(t)$. Consequently, we are motivated to examine travelling wave solutions of the Porous-Fisher-Stefan model and to determine the relationship between $c$ and $\kappa$. To do this, we define $\phi(x, t)=[u(x, t)]^{2}$ to obtain a corresponding PDE with a linear Stefan-like condition at $x=L(t)$ :

$$
\begin{gathered}
\partial_{t} \phi(x, t)=2 \phi(x, t)[1-\sqrt{\phi(x, t)}]+\sqrt{\phi(x, t)} \partial_{x x} \phi(x, t), \quad-\infty<x<L(t), \\
\lim _{x \rightarrow-\infty} \phi(x, t)=1, \quad \phi(L(t), t)=0, \\
\frac{\mathrm{d} L(t)}{\mathrm{d} t}=-\left.\frac{\kappa}{2} \partial_{x} \phi(x, t)\right|_{x \rightarrow L(t)^{-}}, \quad L(0)=L_{0} .
\end{gathered}
$$

To study travelling wave solutions of (6)-(8), we transform the system into travelling wave coordinates via $z=x-L_{0}-c t$, where $z \in(-\infty, 0]$. Noting that when $x=L(t)$, we have $L(t)=L_{0}+c t$, and hence, $\mathrm{d} L(t) / \mathrm{d} t=c$. This change of coordinates gives

$$
\begin{aligned}
& \sqrt{\phi(z)} \phi^{\prime \prime}(z)+c \phi^{\prime}(z)+2 \phi(z)(1-\sqrt{\phi(z)})=0, \quad-\infty<z<0, \\
& \lim _{z \rightarrow-\infty} \phi(z)=1, \quad \phi(0)=0, \quad \lim _{z \rightarrow 0^{-}} \phi^{\prime}(z)=-\frac{2 c}{\kappa},
\end{aligned}
$$


where $^{\prime}=\mathrm{d} / \mathrm{d} z$. It is convenient to write (9) as a system of two first order differential equations:

$$
\begin{aligned}
& \phi^{\prime}(z)=\psi(z) \\
& \psi^{\prime}(z)=-\frac{c \psi(z)}{\sqrt{\phi(z)}}-2 \sqrt{\phi(z)}(1-\sqrt{\phi(z)}) .
\end{aligned}
$$

A general explicit solution of (11)-12 is not obvious, so we seek to study solutions of this system using the $(\phi(z), \psi(z))$ phase plane. There are only two fixed points of (11)-(12): $(0,0)$ and $(1,0)$. Typically, with phase plane analysis of travelling wave solutions, we explore the possibility of a heteroclinic orbit between these fixed points by considering the local behaviour of the linearized system near the fixed points [1, 23]. For the Porous-Fisher model, whose phase plane is identical to (11)-(12), such heteroclinic

orbits can only occur for $c \geq 1 / \sqrt{2}[1,24$. However, for the Porous-Fisher-Stefan model, we have the addition of the Stefan-like condition in (10). Therefore, travelling wave solutions of the Porous-Fisher-Stefan model do not correspond to a heteroclinc orbit between $(0,0)$ and $(1,0)$, but rather a trajectory between $(1,0)$ and another point that is determined by the moving boundary condition in 10 . This particular trajectory, $\psi(\phi ; c)$, corresponds to the travelling wave solution for a given $c$ with $\lim _{z \rightarrow-\infty} \phi(z)=1$, as well as determining $\kappa$ via 10$): \kappa=-2 c / \psi(0 ; c)$.

To determine $\psi(\phi ; c)$, we divide (12) by (11) and obtain

$$
-\frac{\mathrm{d} \psi(\phi)}{\mathrm{d} \phi}=\frac{c}{\sqrt{\phi}}+\frac{2 \sqrt{\phi}(1-\sqrt{\phi})}{\psi(\phi)}, \quad \psi(1)=0, \quad \lim _{\phi \rightarrow 0^{+}} \psi(\phi)=-\frac{2 c}{\kappa} .
$$

As previously mentioned, physical travelling wave solutions in the Porous-Fisher model can only occur when $c \geq 1 / \sqrt{2}[1,24$, and numerical simulations of the Porous-FisherStefan model indicate that all travelling waves have $c<1 / \sqrt{2}$. As a result, we examine (13) in two limiting regimes: $c \rightarrow 0^{+}$and $c \rightarrow 1 / \sqrt{2}^{-}$.

\subsection{Travelling wave solutions for $c \ll 1$}

We first consider the solution of (13) in the limit where $0 \leq c \ll 1$ by expanding $\psi(\phi)$ as a regular perturbation expansion in $c$, i.e. $\psi(\phi)=V_{0}(\phi)+c V_{1}(\phi)+\mathcal{O}\left(c^{2}\right)$. Substituting this expansion into $(13)$ provides

$$
\begin{array}{lll}
\mathcal{O}(1): & -V_{0}(\phi) \frac{\mathrm{d} V_{0}(\phi)}{\mathrm{d} \phi}=2 \sqrt{\phi}(1-\sqrt{\phi}), & V_{0}(1)=0, \\
\mathcal{O}(c): & -V_{1}(\phi) \frac{\mathrm{d} V_{0}(\phi)}{\mathrm{d} \phi}-V_{0}(\phi) \frac{\mathrm{d} V_{1}(\phi)}{\mathrm{d} \phi}=\frac{V_{0}(\phi)}{\sqrt{\phi}}, & V_{1}(1)=0 .
\end{array}
$$

The solution of (14)-15) are

$$
V_{0}(\phi)=-\sqrt{\frac{2}{3}-\frac{8}{3} \phi^{3 / 2}+2 \phi^{2}} \quad \text { and } \quad V_{1}(\phi)=-\frac{1}{V_{0}(\phi)} \int_{\phi}^{1} \sqrt{\frac{2}{3 s}-\frac{8}{3} \sqrt{s}+2 s} \mathrm{~d} s .
$$


Evaluating these expressions at $\phi=0$ gives a two-term approximation for the wave speed $c$ as a function of $\kappa$, provided that $c \ll 1$ :

$$
\kappa \sim \frac{54 \sqrt{3} c}{27 \sqrt{2}-\alpha c} \Longleftrightarrow c \sim \frac{27 \sqrt{2} \kappa}{54 \sqrt{3}+\alpha \kappa},
$$

where $\alpha=36 \sqrt{2}-6 \sqrt{3}+24 \log [(\sqrt{3}-1) /(3 \sqrt{2}-4)] \approx 67.02$. We note that since $\psi=2 u u^{\prime}$ remains $\mathcal{O}(1)$ as $\phi \rightarrow 0^{+}$, this implies that $u^{\prime}=\mathcal{O}\left(u^{-1}\right)$ as $u \rightarrow 0^{+}$, confirming that we are examining a new class of travelling wave solutions with infinite slope at the moving boundary.

\subsection{Travelling wave solutions for $c \rightarrow 1 / \sqrt{2}^{-}$}

From [1, 20, 24], we know that when $c=1 / \sqrt{2}$, (13) has the solution $u^{\prime}=(u-1) / \sqrt{2}$, implying that $\psi=2 u u^{\prime} \rightarrow 0$ as $u \rightarrow 0^{+}$. The Stefan-like condition in (13) requires that $-2 c / \kappa$ must also equal zero. Since $c \neq 0$, this implies that $\kappa \rightarrow \infty$ as $c \rightarrow 1 / \sqrt{2}-$. To determine the leading-order behaviour of $\kappa$ in this limit, we let $c=1 / \sqrt{2}-\varepsilon$, with $0 \leq \varepsilon \ll 1$, and $(13)$ becomes

$$
-\frac{\mathrm{d} \psi(\phi)}{\mathrm{d} \phi}=\frac{1}{\sqrt{2 \phi}}-\frac{\varepsilon}{\sqrt{\phi}}+\frac{2 \sqrt{\phi}(1-\sqrt{\phi})}{\psi(\phi)}, \quad \psi(1)=0, \quad \lim _{\phi \rightarrow 0^{+}} \psi(\phi)=-\frac{2}{\kappa}\left(\frac{1}{\sqrt{2}}-\varepsilon\right) .
$$

We perform a regular perturbation expansion in $\varepsilon$, i.e. $\psi=\Psi_{0}(\phi)+\varepsilon \Psi_{1}(\phi)+\mathcal{O}\left(\varepsilon^{2}\right)$, which gives

$$
\begin{array}{lll}
\mathcal{O}(1): & -\frac{\mathrm{d} \Psi_{0}(\phi)}{\mathrm{d} \phi}=\frac{1}{\sqrt{2 \phi}}+\frac{2 \sqrt{\phi}(1-\sqrt{\phi})}{\Psi_{0}(\phi)}, & \Psi_{0}(1)=0, \\
\mathcal{O}(\varepsilon): & -\Psi_{1}(\phi) \frac{\mathrm{d} \Psi_{0}(\phi)}{\mathrm{d} \phi}-\Psi_{0}(\phi) \frac{\mathrm{d} \Psi_{1}(\phi)}{\mathrm{d} \phi}=\frac{\Psi_{1}(\phi)}{\sqrt{2 \phi}-\frac{\Psi_{0}(\phi)}{\sqrt{\phi}},} & \Psi_{1}(1)=0 .
\end{array}
$$

Noting that $u_{0}^{\prime}=\left(u_{0}-1\right) / \sqrt{2}$ is the leading-order solution in the original variables, the solutions of (19)-20) are

$$
\Psi_{0}(\phi)=2 u_{0} u_{0}^{\prime}=-\sqrt{2 \phi}(1-\sqrt{\phi}) \quad \text { and } \quad \Psi_{1}(\phi)=-\frac{2(1-\sqrt{\phi})}{3} .
$$

Evaluating $\Psi_{0}, \Psi_{1}$ at $\phi=0$ retrieves the Stefan-like condition in (18); hence, for $c=1 / \sqrt{2}-\varepsilon$, corresponding to $\kappa \gg 1$, we have

$$
\kappa \sim \frac{3 \sqrt{2} c}{1-\sqrt{2} c} \Longleftrightarrow c \sim \frac{\kappa}{\sqrt{2}(\kappa+3)} .
$$

In the limit where $\kappa \gg 1$, we can also approximate the travelling wave front $\phi(z)$. To do this, we note that

$$
\frac{\mathrm{d} \phi}{\mathrm{d} z} \sim \Psi_{0}(\phi)+\varepsilon \Psi_{1}(\phi)=\sqrt{2}(\sqrt{\phi}-1)\left(\sqrt{\phi}+\frac{\varepsilon \sqrt{2}}{3}\right), \quad \phi(0)=0,
$$


with solution

$$
z(\phi)=\frac{3 \sqrt{2}}{3+\varepsilon \sqrt{2}}\left[\log (1-\sqrt{\phi})+\frac{\varepsilon \sqrt{2}}{3} \log \left(1+\frac{3}{\varepsilon} \sqrt{\frac{\phi}{2}}\right)\right] .
$$

Equivalently, the original travelling wave $u(z)=\sqrt{\phi(z)}$ can be written implicitly, using 22 , as

$$
z(u) \sim \frac{(\kappa+3) \sqrt{2}}{\kappa+4}\left[\log (1-u)+\frac{\log (1+(\kappa+3) u)}{\kappa+3}\right] .
$$

Thus, we can approximate the sharp moving front $u(z)$, along with its corresponding wave speed $c$, in the regime where $\kappa$ is large. Furthermore, we note that in the limit where $\kappa \rightarrow \infty$, we have $z(u) \rightarrow \sqrt{2} \log (1-u)$, implying that $u(z)=1-\exp (z / \sqrt{2})$. This result agrees with the sharp-fronted travelling wave determined in [1, 20, 24].

\subsection{Comparison of travelling wave solutions}

To validate our asymptotic approximations, we firstly examine the trajectory $\psi(\phi ; c)$, which solves (13) for a given $c$, in the $(\phi, \psi)$-phase plane. To determine $\psi(\phi ; c)$, we solve (13) numerically using ode45 in MATLAB. In Figure 2a, the two-term approximation $V_{0}(\phi)+c V_{1}(\phi)$, valid when $c \ll 1$, agrees very well with $\psi(\phi ; c)$ up to $c=0.2$. Furthermore, in Figure $2 \mathrm{~b}$, we have good agreement between $\psi(\phi ; c)$ and the two-term approximation $\Psi_{0}(\phi)+\varepsilon \Psi_{1}(\phi)$, in the limit when $c=1 / \sqrt{2}-\varepsilon$ up to $\varepsilon=0.2$.

Since the asymptotic approximations accurately describe $\psi(\phi ; c)$ in the phase plane, we now examine the asymptotic relationship between $c$ and $\kappa$. We can estimate $c(\kappa)$ from the numerically-computed $\psi(\phi ; c)$ by noting that $c=-\kappa \psi(0 ; c) / 2$. Alternatively, we can use the numerical solutions of (6)-(8) to determine the speed of the moving front $L(t)$, once the solution has settled to the travelling wave solution (see Appendix A). Figure 3 shows that these two numerical approaches to determine $c(\kappa)$ are indeed equivalent. Furthermore, we see that both asymptotic approximations for $c(\kappa),(17)$ and (22), agree with the numerically-determined $c(\kappa)$ relationship. To minimize the error between $c(\kappa)$ and its asymptotic approximations, we recommend that (17) be used for $\kappa<2$ and $(22)$ be used for $\kappa>20$. For intermediate values of $\kappa$, it appears that a numerical solution is warranted.

Finally, we compare the asymptotic approximation of the moving front $u(z)=$ $u(x-c t)$, shown in (25), with the numerical solution of (6)-(8), after sufficient time has passed such that the travelling wave has formed (Appendix A). Despite the fact that the asymptotic approximation is only expected to match when $\kappa$ is large, we see in Figure $4 a$ that the asymptotic approximation of the shape of the moving fronts, shown implicitly in 25, matches the numerically-determined travelling wave solution very well, even when $\kappa=1$. Furthermore, the asymptotic approximation in 25 agrees well with the numerically-computed travelling wave fronts for a variety of wave speeds (Figure $4 \mathrm{~b}$ ). 

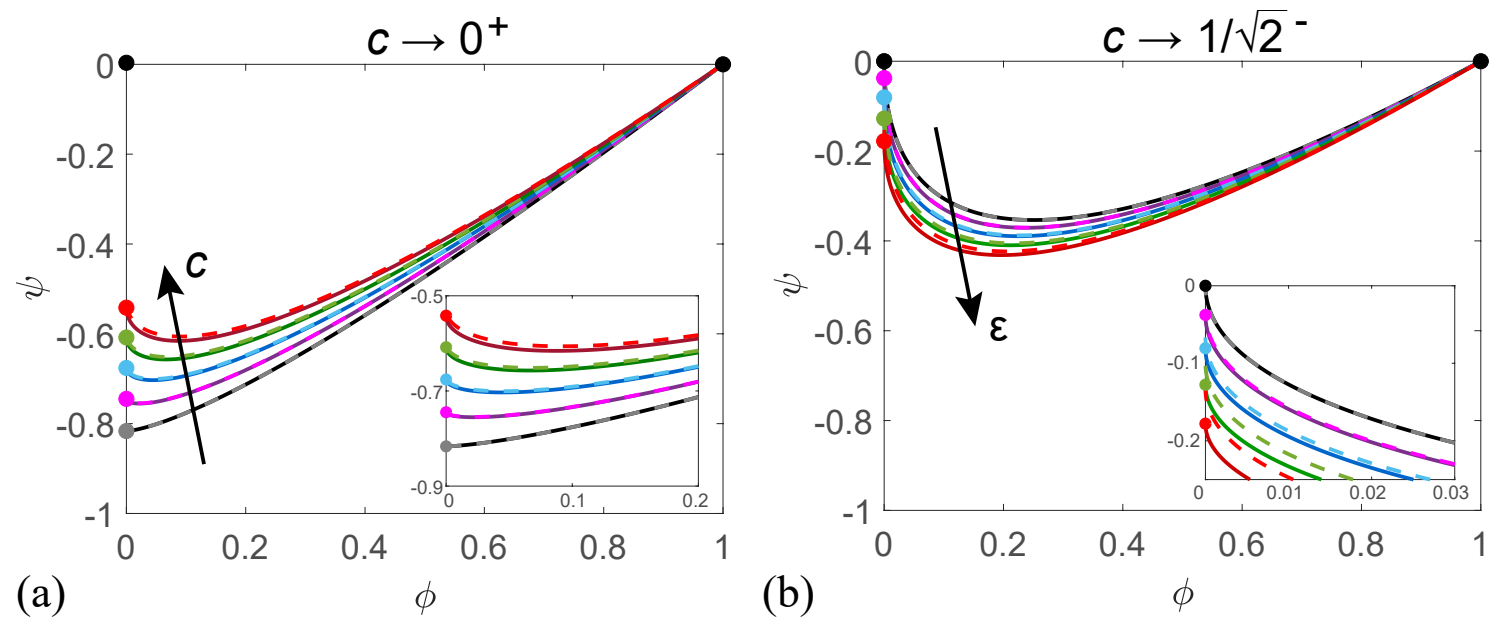

Figure 2. Comparison of the trajectory $\psi(\phi ; c)$ in the $(\phi, \psi)$-phase plane (solid curves), which solves 13 for a given $c$, with its two-term asymptotic approximations (dashed curves). The fixed points $(\phi, \psi)=(0,0),(1,0)$ are shown as black circles, while the Stefan-like condition at $\phi=0$ is shown as coloured circles. (a) The two-term asymptotic approximation when $c \ll 1$ is $V_{0}+c V_{1}$, where $c=0$ (black/grey), $c=0.05$ (pink), $c=0.1$ (blue), $c=0.15$ (green), and $c=0.2$ (red). The black arrow points in the direction of increasing $c$. (b) The two-term asymptotic approximation when $c=1 / \sqrt{2}-\varepsilon$ is $\Psi_{0}+\varepsilon \Psi_{1}$, where $\varepsilon=0$ (black/grey), $\varepsilon=0.05$ (pink), $\varepsilon=0.1$ (blue), $\varepsilon=0.15$ (green), and $\varepsilon=0.2$ (red). The insets in (a) and (b) show the solutions near $\phi=0$. The black arrow points in the direction of increasing $\varepsilon$.

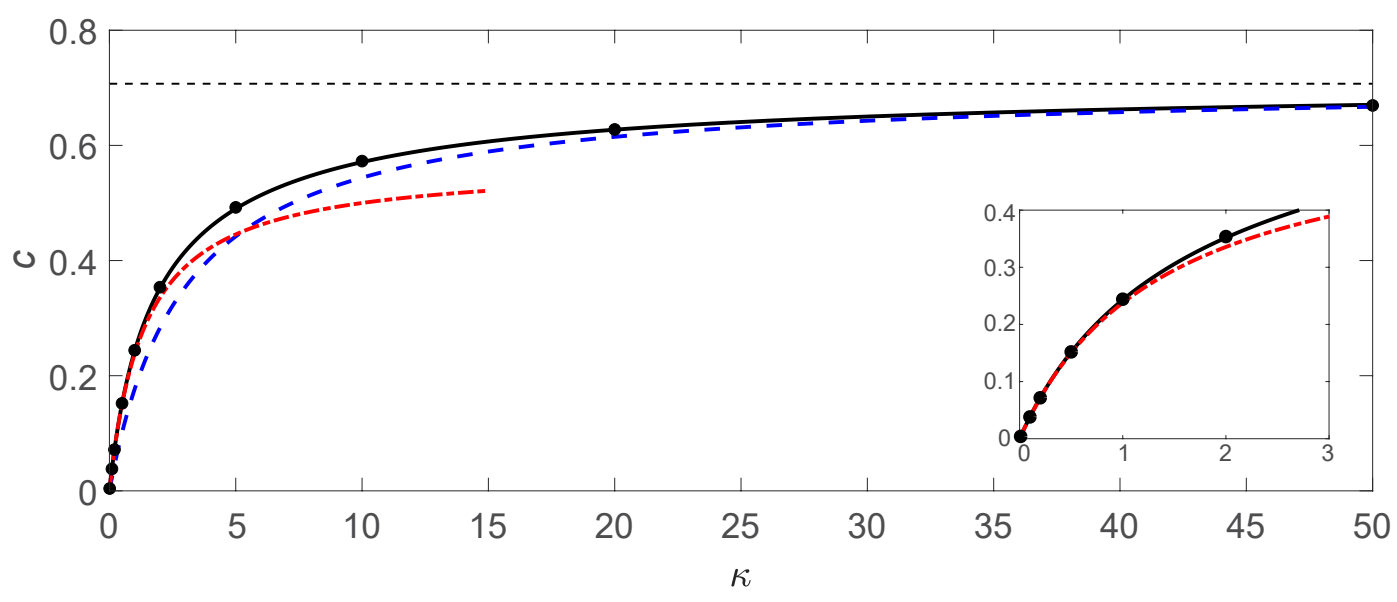

Figure 3. Comparison of the $c(\kappa)$ curves: numerically computed from (6)-(8) (black circles), numerically computed from (13) (black solid curve), and the two-term approximations for $c \ll 1$ (red dot-dash curve) and $\kappa \gg 1$ (blue dashed curve). The critical wave speed $c=1 / \sqrt{2}$ is shown as a black dashed line. 

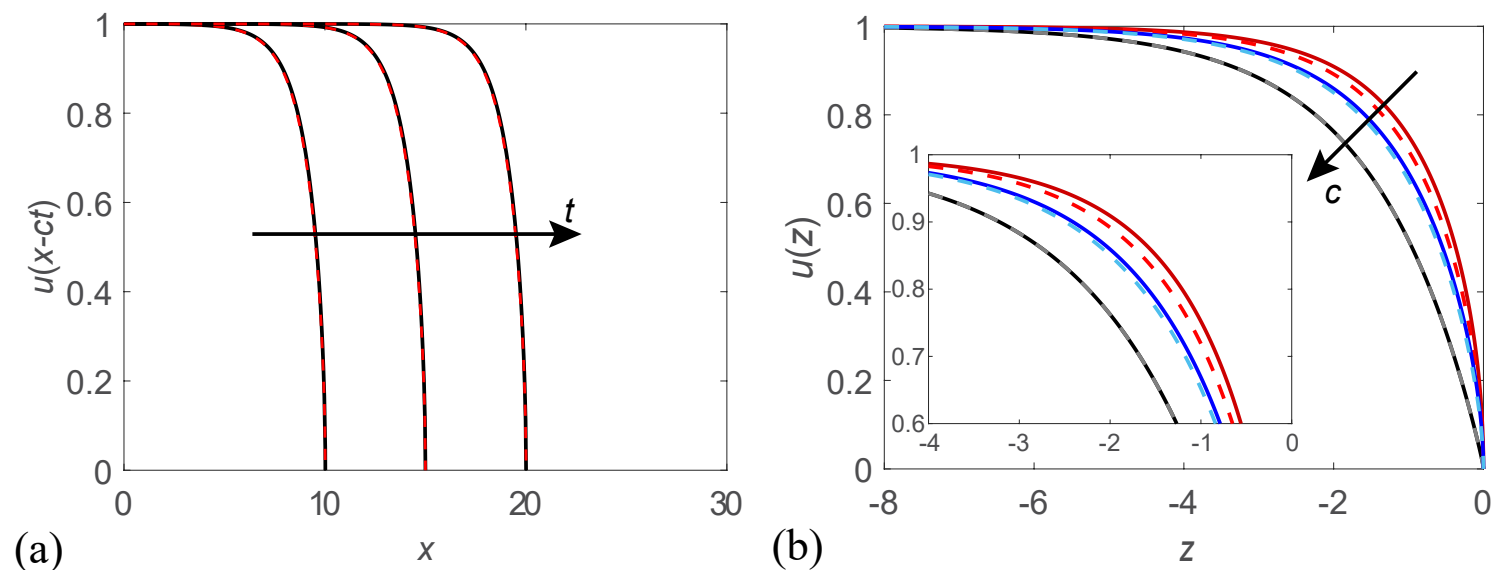

Figure 4. (a) Comparison of the travelling wave front $u(x-c t)$ for $\kappa=1$. The numerical solution of (6)-8) is shown in black at equally-spaced times $(t=$ $41.0,61.5,82.0)$, using (25) as an initial condition. The red-dashed front is determined from large- $\kappa$ asymptotics (25). The black arrow points in the direction of increasing $t$. (b) Travelling wave fronts $u(z)$ (solid curves), superimposed on the corresponding asymptotic approximations (dashed curves) determined using (22) and (25), for $c=0.7$ (black/grey), $c=0.4$ (blue), and $c=0.1$ (red). The corresponding values of $\kappa$ are 287.1 (295.5), 2.711 (3.907), and 0.2958 (0.4941), respectively, where numerically-determined values are shown in the text and asymptotic approximations of $\kappa$ using 22$]$ are shown in parentheses. The black arrow points in the direction of increasing $c$.

\section{Conclusions}

In this work, we consider new travelling wave solutions for the Porous-Fisher model with a moving boundary. This model has certain features that could be considered to be advantageous over other modifications of the Fisher-KPP model. In summary, the new travelling wave solutions: (i) move with a speed that is slower than the more standard Porous-Fisher model; (ii) never lead to population extinction; (iii) have compact support and a well-defined moving front, and (iv) the travelling wave profiles have an infinite slope at the moving front. Using travelling wave coordinates, we transform the model into a single nonlinear differential equation. The solution of this differential equation, corresponding to a particular trajectory in the associated phase plane, determines the travelling wave front and can be approximated using asymptotic analysis in two limiting parameter regimes. In both cases, we obtain a good approximation of this trajectory, which can also be used to relate the speed of the moving front to $\kappa$, a constant appearing in the Stefan-like condition. Finally, we determine a highly-accurate approximate form of the sharp-fronted travelling wave with infinite slope at the moving boundary, corresponding to solutions with wave speeds $0 \leq c<1 / \sqrt{2}$.

Further extensions of this Porous-Fisher-Stefan model can also be made. For instance, the asymptotic analysis performed in this work can be extended to include higher-order terms. Another possible extension could be to consider generalizing the nonlinear degenerate diffusivity function to $D(u)=u^{n}$, for some constant $n>0$ 
[6, 10, 17, 19, 26]. We leave both extensions for future consideration.

\section{Acknowledgments}

This work is supported by the Australian Research Council (DP170100474). The authors thank the two anonymous referees for their helpful comments.

\section{Appendix A. Numerical Solution for the Porous-Fisher-Stefan model}

A MATLAB implementation of the numerical solution of (6)-(8), described below, can be found at https://github.com/nfadai/Fadai_TW2019.

To numerically compute solutions of (6)-(8), we first approximate the semi-infinite domain $(-\infty, L(t)]$ as the finite domain $[0, L(t)]$, provided that $L_{0}>0$. Additionally, we transform (6) -(8) to a fixed-space domain by setting $\xi=x / L(t)$. Consequently,(6)-(8) becomes

$$
\begin{aligned}
& \partial_{t} \phi(\xi, t)=\frac{\xi}{L(t)} \frac{\mathrm{d} L(t)}{\mathrm{d} t} \partial_{\xi} \phi(\xi, t)+2 \phi(\xi, t)[1-\sqrt{\phi(\xi, t)}]+\frac{\sqrt{\phi(\xi, t)}}{[L(t)]^{2}} \partial_{\xi \xi} \phi(\xi, t), \\
& \left.\partial_{\xi} \phi(\xi, t)\right|_{\xi=0}=0, \quad \phi(1, t)=0, \quad 0<\xi<1, \\
& L(t) \frac{\mathrm{d} L(t)}{\mathrm{d} t}=-\left.\frac{\kappa}{2} \partial_{\xi} \phi(\xi, t)\right|_{\xi=1}, \quad L(0)=L_{0} .
\end{aligned}
$$

To compute the numerical solution of (A.1) $-\left(\right.$ A.3), we must specify values for $L_{0}, \kappa$ and $\phi(\xi, 0)$. We obtain numerical solutions of (A.1) on a uniformly-spaced mesh of $\xi \in[0,1]$, i.e. $\xi_{i}=i \Delta \xi, i=0, \ldots, N$, where $\Delta \xi=1 / N$. We denote $\phi\left(\xi_{i}, t_{j}\right)=\phi_{i}^{n}$ and $L\left(t_{j}\right)=L_{j}$ for convenience, where $n \geq 1$ is the $n$th Picard iteration estimate at time $t_{j}$. Therefore, to determine $\phi_{i}$, we use

$$
\begin{aligned}
\frac{\phi_{i}^{n}-\phi_{i}^{p}}{\Delta t}= & \frac{\xi_{i}\left(L_{j}-L_{j-1}\right)\left(\phi_{i+1}^{n}-\phi_{i-1}^{n}\right)}{2 L_{j} \Delta t \Delta \xi}+2 \phi_{i}^{n}\left[1-\sqrt{\phi_{i}^{n-1}}\right] \\
& +\frac{\left(\phi_{i+1}^{n}-2 \phi_{i}^{n}+\phi_{i-1}^{n}\right) \sqrt{\phi_{i}^{n-1}}}{\left(L_{j}\right)^{2}(\Delta \xi)^{2}} \\
& \phi_{0}^{n}=\phi_{1}^{n}, \quad \phi_{N}^{n}=0 .
\end{aligned}
$$

Here, $\phi_{i}^{p}$ is the solution of $\phi_{i}$ at the previous timestep, $t_{j-1}$, and $\Delta t$ is the timestep. We identify the system A.4 A.5 as a tridiagonal matrix in $\phi_{i}^{n}$ at time $t_{j}$, which we can solve efficiently using the Thomas algorithm. This solution is stored as $\boldsymbol{\Phi}_{n}$; if $\max \left|\Phi_{n}-\Phi_{n-1}\right|<\delta$, where $\delta$ is some user-specified tolerance, then the Picard loop terminates and we proceed to updating the moving boundary for the next timestep. Otherwise, $n=n+1$, the solution $\boldsymbol{\Phi}_{n}$ is stored as $\phi_{i}^{n-1}$, and the Picard iteration loop is performed again. 
From the fixed-boundary PDE, the Stefan-like condition at $\xi=1$ is

$$
L(t) \frac{\mathrm{d} L(t)}{\mathrm{d} t}=-\left.\frac{\kappa}{2} \partial_{\xi} \phi(\xi, t)\right|_{\xi=1}, \quad t \in\left[t_{j}, t_{j+1}\right], \quad L\left(t_{j}\right)=L_{j} .
$$

We approximate $\phi(\xi, t)$ as $\phi\left(\xi, t_{j}\right)$ during the small interval $t \in\left[t_{j}, t_{j+1}\right]$, allowing us to explicitly solve A.6 to give a closed form approximation for $L(t)$ :

$$
L(t)=\sqrt{\left(L_{j}\right)^{2}-\left.\kappa \partial_{\xi} \phi\left(\xi, t_{j}\right)\right|_{\xi=1}\left(t-t_{j}\right)}, \quad t_{j} \leq t \leq t_{j+1} .
$$

Therefore, evaluating this expression at $t=t_{j+1}$ and using a second-order finite difference approximation of $\left.\partial_{\xi} \phi\left(\xi, t_{j}\right)\right|_{\xi=1}$, we obtain the approximation

$$
L_{j+1}=\sqrt{\left(L_{j}\right)^{2}-\frac{\kappa \Delta t\left(3 \phi_{N}^{n}-4 \phi_{N-1}^{n}+\phi_{N-2}^{n}\right)}{2 \Delta \xi}} .
$$

With these updated values of $L_{j+1}$ and $\boldsymbol{\Phi}_{n}=\phi_{i}^{p}$, we update $t=t+\Delta t$ and $j=j+1$; we then repeat the computation to integrate through the next time increment. The algorithm terminates when $t+\Delta t>t_{f}$, where $t_{f}$ is the user-specified final time. Once sufficient time has passed that the solution settles towards a travelling wave, we expect that $\mathrm{d} L(t) / \mathrm{d} t=c$, so we fit a straight line to our numerical estimate of $L(t)$ and use the slope of that line to provide an estimate of $c$. 


\section{References}

[1] Murray JD. Mathematical Biology I: An Introduction. Spring-Verlag; 2003.

[2] Fisher RA. The wave of advance of advantageous genes. Annals of Eugenics. 1937;7(4):355-369.

[3] Holmes EE, Lewis MA, Banks JE, Veit RR. Partial differential equations in ecology: spatial interactions and population dynamics. Ecology. 1994;75(1):17-29.

[4] Bao W, Du Y, Lin Z, Zhu H. Free boundary models for mosquito range movement driven by climate warming. Journal of Mathematical Biology. 2018;76(4):841-875.

[5] Sherratt JA, Murray JD. Models of epidermal wound healing. Proceedings of the Royal Society B: Biological Sciences. 1990;241(1300):29-36.

[6] McCue SW, Jin W, Moroney TJ, Lo KY, Chou SE, Simpson MJ. Hole-closing model reveals exponents for nonlinear degenerate diffusivity functions in cell biology. Physica D: Nonlinear Phenomena. 2019;398:130-140.

[7] El-Hachem M, McCue SW, Jin W, Du Y, Simpson MJ. Revisiting the FisherKolmogorov-Petrovsky-Piskunov equation to interpret the spreading-extinction dichotomy. Proceedings of the Royal Society A: Mathematical, Physical and Engineering Sciences. 2019;475(20190378).

[8] Maini PK, McElwain DLS, Leavesley DI. Travelling waves in a wound healing assay. Applied Mathematics Letters. 2004;17(5):575-580.

[9] Maini PK, McElwain DLS, Leavesley DI. Traveling wave model to interpret a wound-healing cell migration assay for human peritoneal mesothelial cells. Tissue Engineering. 2004;10(3-4):475-482.

[10] Simpson MJ, Landman KA, Hughes BD, Newgreen DF. Looking inside an invasion wave of cells using continuum models: proliferation is the key. Journal of Theoretical Biology. 2006;243(3):343-360.

[11] Simpson MJ, Baker RE, McCue SW. Models of collective cell spreading with variable cell aspect ratio: a motivation for degenerate diffusion models. Physical Review E. 2011;83(021901).

[12] McGuinness MJ, Please CP, Fowkes N, McGowan P, Ryder L, Forte D. Modelling the wetting and cooking of a single cereal grain. IMA Journal of Management Mathematics. 2000;11(1):49-70.

[13] Dalwadi MP, O'Kiely D, Thomson SJ, Khaleque TS, Hall CL. Mathematical modeling of chemical agent removal by reaction with an immiscible cleanser. SIAM Journal on Applied Mathematics. 2017;77(6):1937-1961.

[14] Fadai NT, Please CP, Van Gorder RA. Asymptotic analysis of a multiphase drying model motivated by coffee bean roasting. SIAM Journal on Applied Mathematics. 2018;78(1):418-436. 
[15] Brosa Planella F, Please CP, Van Gorder RA. Extended Stefan problem for solidification of binary alloys in a finite planar domain. SIAM Journal on Applied Mathematics. 2019;79(3):876-913.

[16] Treloar KK, Simpson MJ. Sensitivity of edge detection methods for quantifying cell migration assays. PloS One. 2013;8(6):e67389.

[17] Aronson DG. Density-dependent interaction-diffusion systems. In: Dynamics and modelling of reactive systems. Elsevier; 1980. p. 161-176.

[18] Harris S. Fisher equation with density-dependent diffusion: special solutions. Journal of Physics A: Mathematical and General. 2004;37(24):6267.

[19] Gilding BH, Kersner R. A Fisher/KPP-type equation with density-dependent diffusion and convection: travelling-wave solutions. Journal of Physics A: Mathematical and General. 2005;38(15):3367.

[20] Sánchez Garduno F, Maini PK. An approximation to a sharp type solution of a density-dependent reaction-diffusion equation. Applied Mathematics Letters. 1994;7(1):47-51.

[21] Witelski TP. Shocks in nonlinear diffusion. Applied Mathematics Letters. 1995;8(5):27-32.

[22] Witelski TP. Merging traveling waves for the porous-Fisher's equation. Applied Mathematics Letters. 1995;8(4):57-62.

[23] Sánchez Garduno F, Maini PK. Traveling wave phenomena in some degenerate reaction-diffusion equations. Journal of Differential Equations. 1995;117(2):281319.

[24] Sherratt JA, Marchant BP. Nonsharp travelling wave fronts in the Fisher equation with degenerate nonlinear diffusion. Applied Mathematics Letters. 1996;9(5):33-38.

[25] Du Y, Lin Z. Spreading-vanishing dichotomy in the diffusive logistic model with a free boundary. SIAM Journal on Mathematical Analysis. 2010;42(1):377-405.

[26] Wang DS, Zhang ZF. On the integrability of the generalized Fisher-type nonlinear diffusion equations. Journal of Physics A: Mathematical and Theoretical. 2008;42(3):035209. 